\title{
The high-conductance state enables neural sampling in networks of LIF neurons
}

\author{
Mihai A Petrovici ${ }^{*}$, Ilja Bytschok ${ }^{1}$, Johannes Bill², Johannes Schemmel ${ }^{1}$, Karlheinz Meier ${ }^{1}$ \\ From 24th Annual Computational Neuroscience Meeting: CNS*2015 \\ Prague, Czech Republic. 18-23 July 2015
}

The apparent stochasticity of in-vivo neural circuits has long been hypothesized to represent a signature of ongoing stochastic inference in the brain [1-3]. More recently, a theoretical framework for neural sampling has been proposed, which explains how sample-based inference can be performed by networks of spiking neurons $[4,5]$. One particular requirement of this approach is that the membrane potential of these neurons satisfies the socalled neural computability condition (NCC), which in turn leads to a logistic neural response function.

Analytical approaches to calculating this function have been the subject of many theoretical studies. In order to make the problem tractable, particular assumptions regarding the neural or synaptic parameters are usually made [6,7]. However, biologically significant activity regimes exist which are not covered by these approaches: Under strong synaptic bombardment, as is often the case in cortex, the neuron is shifted into a high-conductance state (HCS), which is characterized by a small membrane time constant. In this regime, synaptic time constants and refractory periods dominate membrane dynamics.

The HCS is also particularly interesting from a functional point of view. In [5], we have shown that LIF neurons that are shifted into a HCS by background synaptic bombardment can attain the correct firing statistics to sample from well-defined probability distributions (i.e., satisfy the NCC). In order to calculate the response function of neurons in this regime, we are required to consider a new approach.

The core idea of this approach is to separately consider two different "modes" of spiking dynamics: burst spiking and transient quiescence, in which the neuron does not spike for longer periods. For the bursting mode, we explicitly take into consideration the autocorrelation of the

\footnotetext{
* Correspondence: mpedro@kip.uni-heidelberg.de

${ }^{1}$ Kirchhoff-Institute for Physics, University of Heidelberg, Heidelberg, Germany Full list of author information is available at the end of the article of the article
}

membrane potential before and after refractoriness by propagating the PDF of the effective membrane potential from spike to spike within a burst. For the membrane potential evolution between bursts, we consider an Ornstein-Uhlenbeck approximation. We find that our theoretical prediction of the neural response function closely matches simulation data. Moreover, in the HCS scenario, we show that the neural response function becomes symmetric and can be well approximated by a logistic function, thereby providing the correct dynamics in order to perform neural sampling. Such stochastic firing units can then be used to sample from arbitrary probability distributions over binary random variables $[4,5,8,9]$. We hereby provide not only a normative framework for Bayesian inference in cortex, but also powerful applications of low-power, accelerated neuromorphic systems to highly relevant machine learning problems.

\section{Acknowledgements}

This research was supported by EU grants \#269921 (BrainScaleS), \#237955 (FACETS-ITN), \#604102 (Human Brain Project), the Austrian Science Fund FWF \#1753-N23 (PNEUMA) and the Manfred Stärk Foundation.

\section{Authors' details}

${ }^{1}$ Kirchhoff-Institute for Physics, University of Heidelberg, Heidelberg, Germany. ${ }^{2}$ Institute for Theoretical Computer Science, University of Graz, Graz, Austria.

\section{Published: 18 December 2015}

\section{References}

1. Körding K, Wolpert D: Bayesian integration in sensorimotor learning. Nature 2004, 427:244-247.

2. Fizser J, Berkes P, Orbán G, Lengyel M: Statistically optimal perception and learning: from behavior to neural representations. Trends in Cognitive Sciences 2010, 14(3):119-130.

3. Friston $\mathrm{K}$, Mattout J, Kilner J: Action understanding and active inference. Biological Cybernetics 2011, 104(1-2):137-160.

4. Büsing Lars, Bill Johannes, Nessler Bernhard, Maass Wolfgang: Neural dynamics as sampling: A model for stochastic computation in recurrent networks of spiking neurons. PLoS Computational Biology 2011, 7(11): e1002211.
C Biomed Central 
5. Petrovici MA, Bill J, Bytschok I, Schemmel J, Karlheinz Meier: Stochastic inference with deterministic spiking neurons. arXiv preprint 2013,

1311.3211.

6. Brunel N, Sergi S: Firing Frequency of leaky integrate-and-fire neurons with synaptic current dynamics. Journal of Theoretical Biology 1998, 195(1):87-95.

7. Moreno-Bote R, Parga N: Role of synaptic filtering on the firing response of simple model neurons. Physical Review Letters 2004, 92:028102.

8. Pecevski D, Büsing L, Maass W: Probabilistic inference in general graphical models through sampling in stochastic networks of spiking neurons. PLoS Computational Biology 2011, 7(12):e1002294.

9. Probst D, Petrovici MA, Bytschok I, Bill J, Peceyski D, Schemmel J, Meier K: Probabilistic inference in discrete spaces can be implemented into networks of LIF neurons. Frontiers in Neuroscience 2015, 9:13.

doi:10.1186/1471-2202-16-S1-O2

Cite this article as: Petrovici et al:: The high-conductance state enables neural sampling in networks of LIF neurons. BMC Neuroscience 2015 16(Suppl 1):O2.

\section{Submit your next manuscript to BioMed Central} and take full advantage of:

- Convenient online submission

- Thorough peer review

- No space constraints or color figure charges

- Immediate publication on acceptance

- Inclusion in PubMed, CAS, Scopus and Google Scholar

- Research which is freely available for redistribution

Submit your manuscript at www.biomedcentral.com/submit 